SORDI STOCK, Bárbara. "Programas de rehabilitación para agresores en España: un elemento indispensable de las políticas del combate a la violencia de género".

Polít. crim. Vol. 10, No 19 (Julio 2015), Art. 10, pp. 297-317.

[http://www.politicacriminal.cl/Vol_10/n_19/Vol10N19A10.pdf]

\title{
Programas de rehabilitación para agresores en España: un elemento indispensable de las políticas de combate a la violencia de género.
}

\section{Rehabilitation programs for aggressors in Spain: an indispensable element of policies to combat gender violence.}

\author{
Bárbara Sordi Stock \\ Doctora en Derecho (Mención Internacional) \\ Grupo de Investigación Criminología y Derecho Penal. Instituto Andaluz Interuniversitario \\ de Criminología - Universidad de Sevilla (España) \\ barbarasordistock@gmail.com
}

\section{Resumen}

En el presente estudio se sintetizan los principales hallazgos publicados en España sobre los programas de rehabilitación para agresores de violencia de género como pena/ medida alternativa a la prisión. España ha experimentado un cambio jurídico radical en el enfrentamiento de la violencia hacia la mujer pareja o ex pareja a lo largo de los últimos diez años, incorporando paulatinamente los programas como respuesta penal a esta clase de delitos. Paralelamente, un amplio número de investigaciones para identificar los resultados preventivos especiales se han llevado a cabo, labor que ha dado pié a la creación de una importante base de datos para los países con menor tradición rehabilitadora, como los latinoamericanos. Si bien los desafíos siguen siendo de los más diversos órdenes, los hallazgos empíricos revelan que los programas son un elemento indispensable de las políticas de combate a la violencia de género en razón de los efectos preventivos especiales que presentan. A día de hoy la discusión se centra en cómo rehabilitar y no si es posible rehabilitar.

Palabras clave: violencia de género, programas de rehabilitación, penas y medidas alternativas a la prisión.

\begin{abstract}
The aim of this study is to summarize the scientific knowledge about rehabilitation programs to gender violence developed in Spain. Over the last ten years Spain has experienced a radical legal change in strategies to deal with violence against women and has gradually incorporated programs as a criminal response to such crimes. In parallel, a large number of research to identify preventive results have been carried out, work that has given rise to the creation of an important database for countries with a lesser rehabilitative tradition, such as those in Latin America. While an ample variety of challenges remain, empirical findings reveal that these programs are an essential element of policies to combat gender violence because of the special preventive effects they present. The discussion today is focused on how to rehabilitate and not whether it is possible to rehabilitate.
\end{abstract}


SORDI STOCK, Bárbara. "Programas de rehabilitación para agresores en España: un elemento indispensable de las políticas del combate a la violencia de género".

Key words: gender violence, batterers' intervention program, community sentences.

\section{Introducción.}

España ha experimentado un cambio jurídico radical en el enfrentamiento de la violencia hacia la mujer pareja o ex pareja. Entre las medidas gubernamentales para reducir el impacto de este problema social se encuentran los programas de rehabilitación para agresores vinculados a la Justicia Penal. Estos han sido particularmente impulsados por la Ley Orgánica 1/2004, de 28 de diciembre, de Medidas de Protección Integral contra la Violencia de Género (en adelante LO 1/2004), que determina que la Administración Penitenciara realice intervenciones específicas dentro de prisiones para reclusos por crímenes de esta naturaleza (art. 42). Fuera de prisiones, los programas ganan paulatinamente nueva configuración; han pasado a ser reglas de conductas obligatorias asociadas a la suspensión de la pena privativa de libertad (art. 83 del Código Penal español, en adelante CP) y a la sustitución de la pena de prisión (art. $88 \mathrm{CP}$ ) y, más recientemente, previstos como modalidad de Trabajos en Beneficio de la Comunidad (art. 49 CP).

Como no podría ser de otra forma, al saber científico le corresponde la función, irrenunciable, de evaluar los programas ejecutados. En otras palabras, los cambios legales y en la Administración de la Justicia ocurridos a lo largo de los últimos diez años solo adquieren legitimidad si la evidencia científica demuestra que es posible rehabilitar al agresor. Un amplio número de expertos no escatiman esfuerzos para apurar los resultados alcanzados, sirviendo los hallazgos de motor para el incremento de la confianza en esta respuesta penal. Los programas comunitarios ganan tal visibilidad que, a día de hoy, la Dirección General de Instituciones Penitenciarias/Ministerio del Interior ha afianzado su posición en el sentido de que todos los agresores por violencia de género deben participar necesariamente de un programa específico vinculado a la Justicia Penal.

Los países con menor tradición rehabilitadora, como los latinoamericanos, fijan su mirada en el otro lado del Atlántico a fin de aclarar si las evidencias de que se dispone en España justifican que los programas constituyan un elemento clave de las políticas penales de combate a la violencia de género. El presente artículo busca contribuir al desate de esta cuestión. A continuación se sintetizarán los principales hallazgos publicados en España en el ámbito de los programas de rehabilitación como pena/ medida alternativa a la prisión. El análisis resulta interesante porque algunos de los entrabes ahí encontrados se repiten en aquellos países cuyos programas vinculados a la Justicia Penal están en fase inicial.

\section{1. ¿Qué dice la evidencia científica sobre los programas de rehabilitación?}

\subsection{Diseño del programa: enfoque, extensión y formato grupal/individual.}

En España se han ejecutado (y se siguen ejecutando) programas con distintos diseños. Desde la Justicia Penal, el año de 2004 supuso un punto de inflexión, ya que con la entrada en vigor de la LO 1/2004 se iniciaron los programas de cumplimiento obligatorio para los 
Polít. crim. Vol. 10, № 19 (Julio 2015), Art. 10, pp. 297-317.

[http://www.politicacriminal.cl/Vol_10/n_19/Vol10N19A10.pdf]

casos de medidas alternativas a la prisión (art. 83 y $88 \mathrm{CP})^{1}$. En la práctica, las intervenciones se han configurado de una manera muy híbrida sin que concurra una apuesta estatal para su promoción, estructuración y coordinación ${ }^{2}$. Esta realidad da un giro a partir de 2010, cuando el Estado adopta una estrategia más clara sobre cómo deben ser las mismas. Se inicia un proceso de unificación, resultado de la puesta en marcha por Instituciones Penitenciarias de un Programa Marco titulado Violencia de Género: Programa de Intervención para Agresores $^{3}$ (en adelante PRIA) y de la creación y reglamentación de una estructura para su ejecución (Real Decreto 840/2011) por medio de los Servicios de Gestión de Penas y Medidas Alternativas (en adelante SGMPA). El PRIA es implantado en todo el territorio nacional, a excepción de la Comunidad Autónoma de Cataluña que cuenta con competencia autónoma en esfera penitenciaria, y está diseñado bajo el enfoque cognitivo-conductual con perspectiva de género, en 25 sesiones grupales, de 2h30min de duración una vez a la semana, a lo largo de 6 meses.

Particularmente sobre el enfoque, entre los programas españoles se han encontrado, entre otros, el cognitivo-conductual ${ }^{4}$, el ecológico ${ }^{5}$ y el cognitivo-conductual con perspectiva de género ${ }^{6}$. La actual preferencia por el último no se debe al fracaso de las experiencias anteriores, sino es resultado del trabajo de profesionales que lo juzgaron como fundamental $^{7}$. Justamente, el PRIA presenta como características diferenciales la mezcla de aspectos clínicos y la perspectiva de género, el énfasis en la motivación inicial y el análisis de las distintas conductas que integran la violencia. Parte de la comunidad científica ha interpretado el enfoque de género, que asume el patriarcado como principal factor explicativo de la violencia, como muy positivo ${ }^{8}$. Destacan que las creencias mantenidas

\footnotetext{
${ }^{1}$ Para un análisis en profundidad sobre la configuración jurídica de los programas como pena/ medida alternativa a la prisión consultar SORDI STOCK, Bárbara, "¿Nuevos horizontes? en los programas de rehabilitación para agresores de violencia de género", InDret - Revista para el análisis del Derecho, 1/2015.

${ }^{2}$ BOIRA SARTO, Santiago, Hombres Maltratadores. Historias de Violencia Masculina, Zaragoza: Prensas Universitarias de Zaragoza, 2010.

${ }^{3}$ SECRETARIA GENERAL DE INSTITUCIONES PENITENCIARIAS, Documentos Penitenciarios 7. Violencia de Género. Programa de Intervención para Agresores - PRIA, Madrid: 2010. Disponible en http://www.institucionpenitenciaria.es/ [visitado el 08.04.2015].

${ }^{4}$ SECRETARIA GENERAL DE INSTITUCIONES PENITENCIARIAS, Documentos Penitenciarios 2. Programa de Tratamiento en Prisión para agresores en el ámbito familiar. Grupo de trabajo sobre violencia de género, Madrid: 2005. Disponible en http://www.institucionpenitenciaria.es/[visitado el 08.04.2015]

${ }^{5}$ LILA, Marisol; GARCÍA, António; LORENZO, María Victória, Manual de Intervención con Maltratadores, Valencia: Universitat de València, 2010.

${ }^{6}$ SECRETARIA GENERAL DE INSTITUCIONES PENITENCIARIAS, Documentos Penitenciarios 7, cit. nota $\mathrm{n}^{\circ} 1$.

${ }^{7}$ Entre otros autores, RUIZ, Sergio; EXPÓSITO, Francisca, "Intervención con hombres en suspensión condicional de condena por violencia de género", Anuario de Psicología Jurídica, Vol. 18 (2008), pp. 81 - 89. TEJERINA, Benjamín; MARTÍNEZ, María, Evaluación de la implementación de programas de intervención re-educativa con agresores en el ámbito de la violencia de género, Informes del Centro de Estudios sobre la Identidad Colectiva - C.E.I.C/IKI: 2011. La práctica del Centro Penitenciario de Albolote - Granada se encuentra entre los gérmenes de la transición al enfoque de género. Se propuso una nueva intervención al entender que el Programa de Tratamiento en Prisión para Agresores en el Ámbito Familiar, anteriormente recomendado por Instituciones Penitenciarias, partía de una mirada excesivamente clínica y, por tanto, necesitaba de una orientación de género.

${ }^{8}$ EXPÓSITO, Francisca; RUIZ, Sergio, "Reeducación de Maltratadores: Una Experiencia de Intervención desde la Perspectiva de Género", Intervención Psicosocial, Vol. 19, nº 2 (2010), pp. 145-151.
} 
SORDI STOCK, Bárbara. "Programas de rehabilitación para agresores en España: un elemento indispensable de las políticas del combate a la violencia de género".

acerca del maltrato son compartidas por muchos hombres que participan de un programa en medio comunitario, circunstancias que potencian la resistencia a la intervención, la falta de conciencia del daño provocado y el victimismo por parte del agresor. En otras palabras, si bien es cierto que quizás no se logre comprobar científicamente los resultados del planteamiento de género, también es cierto que impregna favorablemente el programa. Desde una postura más crítica, algunos investigadores alertan de los riesgos de obviar los aspectos clínicos en el tratamiento del agresor. Concretamente, el de instaurarse un discurso reduccionista sobre las causas y el enfrentamiento de la violencia, cimentándose los programas apenas en el plano ideológico?.

Lo cierto es que en España no se dispone de datos sobre el impacto del enfoque de género en la rehabilitación del agresor. En todo caso, perspectiva de género no es una o dos unidades en el conjunto que conforma la intervención. La propuesta es que, junto a los aspectos clínicos, a lo largo de todo el programa se trabaje con la construcción de las masculinidades y feminidades y su relación con el uso de la violencia como estrategia válida para resolver conflictos; sea la violencia perpetrada por los hombres, sea por las mujeres ${ }^{10}$. En lo relativo a la extensión y formato tampoco está del todo claro los beneficios reales de un programa más largo o del formato grupal sobre el individual. La verdad es que la pluralidad de programas ejecutados ha permitido interesantes propuestas para evaluar el diseño (formato individual/grupal, extensión, enfoque etc.) y su relación con los resultados del tratamiento, con destaque para la predisposición al cambio, aspectos del funcionamiento psicológico relacionados con la dinámica violenta y reincidencia ${ }^{11}$.

De esta forma, si bien la orientación va en la línea de aplicar el Programa Marco PRIA, las ventajas y desventajas sobre los diferentes diseños aún se encuentran en pleno debate ${ }^{12}$. Antes que contraproducente, dicho escenario ha favorecido que la comunidad científica profundice los estudios que tomen en cuenta estas cuestiones y que algunos temas considerados tabús sean tratados con mayor rigor científico ${ }^{13}$. Aquí vale destacar las consecuencias del maltrato para el agresor, o sea, que el haber ejercido violencia de género

\footnotetext{
${ }^{9}$ LOINAZ CALVO, Ismael, Aproximación teórica y empírica al estudio de las tipologías de agresores de pareja: análisis descriptivo de variables e instrumentos de evaluación en el centro penitenciario Brians-2, Madrid: Ministerio del Interior, Secretaria General Técnica, 2009. LOINAZ CALVO, Ismael; ECHEBURÚA, Enrique, "Necesidades terapéuticas en agresores de pareja según su perfil diferencial", Clínica Contemporánea, Vol. 1, no 2 (2010), pp. 85-95.

${ }^{10}$ SORDI STOCK, Bárbara, Análisis político criminal de los programas de rehabilitación para agresores de violencia de género, Tesis Doctoral bajo supervisión de Borja Mapelli Caffarena, Universidad de Sevilla, Departamento de Derecho Penal y Procesal, 2014, pp. 450 y ss.

${ }^{11}$ ECHEBURÚA, Enrique et al., "Evaluación de la eficacia de un tratamiento cognitivo-conductual para hombres violentos contra la pareja en un marco comunitario: Una experiencia de 10 años (1997-2007)", International Journal of Clinical and Health Psychology, $\mathrm{n}^{\circ} 9$ (2009), pp. 199-217. BOIRA SARTO, Santiago et al., "Intervención psicológica en la comunidad en hombres condenados por violencia de género", Anales de Psicología, Vol. 29, nº 1 (2013), pp.19-28.

${ }_{12}$ Véanse los estudios de BOIRA SARTO, Santiago et al., "Evaluación cualitativa de un programa de intervención psicológica con hombres violentos dentro de la pareja.", Acciones e Investigaciones Sociales, Vol. 28 (2010), pp. 135-156 y de ECHEBURÚA, Enrique, "Adherencia al tratamiento en hombres maltratadores contra la pareja en un entorno comunitario: Realidad actual y retos de futuro", Psychosocial Intervention, $\mathrm{n}^{\mathrm{o}} 22$ (2013), pp. 87-93.

${ }^{13}$ ECHEBURÚA, “Evaluación”, cit. nota no 11. BOIRA, "Intervención”, cit. nota no 11.
} 
Polít. crim. Vol. 10, № 19 (Julio 2015), Art. 10, pp. 297-317.

[http://www.politicacriminal.cl/Vol_10/n_19/Vol10N19A10.pdf]

y encontrarse condenado por este delito influye negativamente también en la salud mental del agresor ${ }^{14}$. En este contexto, la satisfacción de los usuarios del programa pasa a ser considerada. Por lo general, los agresores se han mostrado bastante satisfechos con las intervenciones aunque con distintos formatos. Véase por ejemplo en Alicante, dónde el $85 \%$ de los agresores valoraron positivamente un programa tras su finalización y afirmaron que este les ha servido en sus vidas ${ }^{15}$. En Cataluña, de los 58 agresores que habían participado de una intervención, aproximadamente el $90 \%$ ellos se mostró satisfecho con el programa un año más tarde tras ser finalizado. Las personas satisfechas con el programa también se mostraron más satisfechas con su vida, pareja y trabajo, además de asumir más su responsabilidad frente al delito ${ }^{16}$. Insistimos, por tanto, en el importante papel que desempeñan las entidades responsables de los programas de rehabilitación en la vida de los hombres que ejercen violencia.

\subsection{Características de los participantes.}

Sobre los participantes, se trata preponderantemente de españoles con edad media de 40 años, con características socioeconómicas y psicopatológicas muy diversas ${ }^{17}$. Más de un estudio pone el acento en el progresivo aumento de la población extranjera derivada a los programas españoles, con especial destaque para los hombres procedentes de Latino América $^{18}$. Es más, esta es una diferencia bastante significativa entre la población que participa de los programas en medio cerrado y abierto ${ }^{19}$. Señalamos, pues, que el

14 LÓPEZ SAMANIEGO, Luz, "Salud y condena por violencia de género. (Asistentes a programas formativos de reeducación)”, La Ley Penal: revista de Derecho penal, procesal y penitenciario, n 93 (2012), pp. $94-103$.

${ }^{15}$ MAGRO SERVET, Vicente; HERNÁNDEZ RAMOS, Carmelo; CUELLAR OTÓN, Pablo, “La aplicación de programas formativos de reeducación para condenados por delitos relacionados con la violencia de género, en aplicación de las medidas de suspensión de la ejecución de la pena: el programa de la audiencia provincial de alicante", en: MARTÍNEZ GARCÍA, Elena; VEGAS AGUILAR, Juan Carlos, La ejecución de los trabajos en beneficio de la comunidad por delitos de violencia de género, Tirant Monografías 763, Valencia: Tirant lo Blanch, 2012, pp. 172 - 207, p. 200.

${ }^{16}$ PÉREZ RAMÍREZ, Meritxell; MARTÍNEZ GARCÍA, Marian, "Evaluación de los programas formativos aplicados desde la ejecución penal en la comunidad para delitos de violencia de género", en: CENTRE D' ESTUDIS JURÍDICS I FORMACIÓ ESPECIALITZADA, Intervención con agresores de violencia de género. Evaluación de los programas formativos aplicados desde la ejecución penal en la comunidad para delios de violencia de género. Clasificación de agresores de pareja en prisión. Implicaciones terapéuticas y de gestión del riesgo, Justicia y Sociedad, n 34, Centre d' Estudis Jurídics i Formació Especialitzada: 2011, pp. $11-152$, p. 68 y 74.

${ }^{17}$ PÉREZ/MARTÍNEZ, "Evaluación”, cit. nota no 16. MAGRO/HERNÁNDEZ/CUELLAR, "La aplicación”, cit. nota $\mathrm{n}^{\circ}$ 15. SECRETARIA GENERAL DE INSTITUCIONES PENITENCIARIAS - INSTITUTO DE CIENCIAS FORENSES Y DE LA SEGURIDAD, Evaluación del Programa. "Violencia de Género: programa de intervención para agresores”, en medidas alternativas, Madrid: ca. 2012. Disponible en http://www.institucionpenitenciaria.es/ [visitado el 08.04.2015].

18 LILA, Marisol; CONCHELL, Raquel, "Programa CONTEXTO: programa de Intervención para maltratadores en la provincia de Valencia.", ReCrim (2009), pp.199-215, p. 202. MAGRO/HERNÁNDEZ/CUELLAR, "La aplicación”, cit. nota n 15, p. 199. SECRETARIA GENERAL DE INSTITUCIONES PENITENCIARIAS, Evaluación, cit. nota n ${ }^{\circ} 17$, p. 15.

19 FERNÁNDEZ-MONTALVO, Javier et al., "Violencia de género e inmigración: perfil diferencial de hombres maltratadores nacionales e inmigrantes", Psicología Conductual, Vol. 19, nº 2 (2011), pp. 439-452. ECHAURI TIJERAS, José Antonio, "Efectividad de un programa de tratamiento con inmigrantes que ejercen violencia de género contra la pareja", Psicothema, Vol. 25, nº 1 (2013), pp. 49-54. 
SORDI STOCK, Bárbara. "Programas de rehabilitación para agresores en España: un elemento indispensable de las políticas del combate a la violencia de género".

incremento de los extranjeros en los programas desarrollados como medida alternativa es un fenómeno de magnitud nacional, realidad que viene justificando la adaptación de las intervenciones a la multiculturalidad de la población usuaria ${ }^{20}$.

En lo relativo a las características sociodemográficas y psicopatológicas adoptamos como punto de partida de análisis los datos de la evaluación del PRIA ${ }^{21}$. Desde su puesta en marcha en medio comunitario, el mayor porcentaje de usuarios del programa han sido españoles $(76 \%)$, divorciados/separados $(40,5 \%)$, con graduación escolar $(42,2 \%)$, con situación económica suficiente (52\% tenía trabajo) y condenados por malos tratos ocasionales $(71 \%)$. Gran parte de los agresores presentaron creencias sexistas, se demostraron impulsivos, con bajo control de la ira y no reconocieron, minimizaron o delegaron (a la víctima, al consumo de drogas/alcohol y a los celos) la responsabilidad de los hechos. Los hombres, en su mayoría, no presentaban factores de riesgo asociados a la conducta violenta, como por ejemplo historial delictivo o problemas graves de adicción. Dicho estudio, no obstante, llama la atención sobre la existencia de un "grupo de riesgo", compuesto por una minoría de aquéllos que frecuentaron el programa. Los riegos fueron definidos por distintos factores, como por ejemplo el desempleo (37\%), historial de violencia en la familia de origen $(15,4 \%$ ) y uso de alcohol/droga (el $24 \%$ de los usuarios han reconocido que el delito se produjo bajo los efectos del alcohol). En este pequeño grupo también se incluyó a aquellos que no eran violentos ocasionales, a pesar de participar de un programa en medio abierto. Un dato alarmante cuando se desvela que parte de los hombres $(15 \%)$ reconoció haber actuado de forma agresiva con su pareja en más de 5 ocasiones, a pesar de que en muy pocos casos hubiesen sido condenados anteriormente por un delito contra esta misma víctima $(6,5 \%)$. Los trámites de la separación/divorcio también aparecen como un momento de mayor incidencia del acto violento (31\%).

Un repaso sobre los usuarios de los programas desarrollados en las demás Comunidades Autónomas no difiere en demasía de lo expuesto. Años antes en Cataluña se había sacado a la luz que los usuarios de los programas también tenían poca formación (el 38,6\% tenían grado escolar), aunque con trabajo calificado (73\%) y sin un perfil típico de carrera delincuencial. $^{22}$ Asimismo se diagnosticó un grupo minoritario que indicó haber sido testimonio de violencia familiar (18\%), que cometió los actos de violencia durante la separación/ruptura de la pareja (15\%) y que cuando consumaron la agresión estaban bajo los efectos de drogas (35\%). Ya los datos de Valencia revelan, entre otras cuestiones, una precaria formación escolar de muchos penados (aproximadamente el 53\% tenía estudios

\footnotetext{
${ }^{20}$ ECHAURI, "Efectividad", cit. nota n ${ }^{\circ} 19$. FERNÁNDEZ-MONTALVO, "Violencia de género", cit. nota ${ }^{\circ}$ 19. LILA, Marisol et al., "Cuando las cifras hablan: Programa de rehabilitación para maltratadores e inmigración", ca. 2011 Disponible en http://www.uv.es/contexto/enriquegracia/Investigacion.htm [visitado el 08.04.2015]. SECRETARIA GENERAL DE INSTITUCIONES PENITENCIARIAS, El Delito de Violencia de Género y los Penados Extranjeros anexo al Documentos Penitenciarios 7. Violencia de Género. Programa de Intervención para Agresores. PRIA, Madrid: 2011, Disponible en http://www.institucionpenitenciaria.es/ [visitado el 08.04.2015].

${ }^{21}$ SECRETARIA GENERAL DE INSTITUCIONES PENITENCIARIAS, Evaluación, cit. nota $\mathrm{n}^{\circ} 17$.

${ }^{22}$ PÉREZ/MARTÍNEZ, "Evaluación", cit. nota nº 16.
} 
Polít. crim. Vol. 10, No 19 (Julio 2015), Art. 10, pp. 297-317.

[http://www.politicacriminal.cl/Vol_10/n_19/Vol10N19A10.pdf]

primarios o no tenía estudios). ${ }^{23}$ En Alicante, al tiempo que se precisó un perfil socioeconómico inexistente, se constató que un porcentaje de penados presentaron antecedentes familiares de maltrato $(20 \%) .{ }^{24} \mathrm{En}$ el sur, los datos coinciden de forma general con los ya mencionados, si bien se identificó un alto índice de penados en paro $(47 \%)^{25}$.

Lo que pretendemos aquí destacar es que el hecho de que muchos de los usuarios no presentan carrera delictiva consolidada no significa que no existan una serie de factores de riesgo asociados a la conducta violenta. Entre ellos están la baja formación escolar, el desempleo, la violencia en la familia de origen, el uso de alcohol y drogas, creencias sexistas, dificultad de controlar la ira etc. Además, se ha constatado que la ruptura de la relación de pareja es, en innumerables casos, una circunstancia que desencadena la violencia, así como un porcentaje significativo de víctimas sigue manteniendo relación afectiva con el agresor tras su condena. Es más, los datos empíricos revelan algunas características comunes entre los agresores que frecuentaron un programa de rehabilitación independientemente de la Comunidad Autónoma en la cual este tuvo lugar. Debe destacarse la limitada inteligencia emocional ("máscara de autosuficiencia"), inestabilidad emocional (especialmente en relación al control de la ira y de la ansiedad), conducta autoritaria, presencia de celos excesivos, rigidez en los roles de género, violencia asumida como estrategia válida para hacer frente a los problemas y dificultad en aceptar que su conducta bien es un delito o bien afecta a su esposa/compañera o personas que están a su alrededor, como las hijas y los hijos. ${ }^{26}$

Existen, por tanto, un conjunto de variables psicosociales relevantes a ser trabajadas con los sujetos y que deben cautelosamente ser analizadas desde la interdisciplinaridad para que no se corra el riesgo de objetivar en una única variable (reincidencia) los resultados de una intervención que justamente se propone trabajar desde distintos matices ${ }^{27}$. Precisamente, tratar un agresor en medio abierto no significa que este no pueda presentar limitaciones psicológicas importantes. Los déficits psicológicos son compatibles con la imputabilidad de los agresores y están presentes en muchos de $\operatorname{ellos}^{28}$. De otra parte, se ha demostrado que

\footnotetext{
${ }^{23}$ LILA, Marisol et al., "Predicting Success Indicators of an Intervention Programme for Convicted IntimatePartner Violence Offenders: the Contexto Programme.”, The European Journal of Psychology Applied to Legal Context, Vol. 5, no 1 (2013), pp. 73-95, p. 78.

24 MAGRO/HERNÁNDEZ/CUELLAR, “La aplicación”, cit. nota nº 15, p. 199.

${ }^{25}$ ARRIGONI et al., "Aplicación de un programa terapéutico en hombres violentos contra la pareja", Anuario de Psicología Jurídica, no 23 (2013), pp. 3-9.

${ }^{26}$ CONCHELL DIRANZO, Raquel; LILA MURILlO, Marisol; CATALÁ MIÑANA, Alba, "Cambios psicosociales en un programa de intervención con hombres penados por violencia contra la mujer.", Revista de Psicología de la Universidad de Chile, Vol. 21, n 2 (2012), pp. 159 - 185. ECHEBURÚA, "Evaluación", cit. nota $\mathrm{n}^{\circ}$ 11. ECHEBURÚA, Enrique; AMOR, Pedro Javier, "Perfil psicopatológico e intervención terapéutica con los agresores contra la pareja", Revista Española de Medicina Legal, Vol. 36, n 3 (2010), pp. 117-121. MAGRO/HERNÁNDEZ/CUELLAR, "La aplicación”, cit. nota no 15. PÉREZ/MARTÍNEZ, "Evaluación", cit. nota n' 16.

27 ECHEBURÚA, "Evaluación”, cit. nota no 11. LILA, "Predicting", cit. nota no 23.

${ }^{28}$ ECHEBURÚA/AMOR, "Perfill”, cit. nota $n^{\circ} 26$, p. 118. Téngase en cuenta que la ausencia de trastornos de personalidad o psicopatologías graves son factores comunes de exclusión entre los programas para agresores de violencia de género en España.
} 
SORDI STOCK, Bárbara. "Programas de rehabilitación para agresores en España: un elemento indispensable de las políticas del combate a la violencia de género".

algunos no presentan signos de psicopatología, dato que reabre el debate sobre si todos los hombres deben ser enviados a un programa o a un mismo programa ${ }^{29}$.

\subsubsection{Tipologías.}

La comunidad científica viene insistiendo en los beneficios de una clasificación tipológica de los hombres que ejercen violencia. Una propuesta que ha ganado aceptación es la promocionada por Echeburúa y Amor $^{30}$ con base en el tipo de violencia que se ejerce: si la violencia es expresiva, cuando por ejemplo ocurre cuando el agresor tiene dificultad de controlar los impulsos y expresar afecto, o instrumental, cuando la agresión es planificada y habitualmente no viene acompañada de culpa. Ya Redondo Rodríguez ${ }^{31}$, tras analizar una muestra de 266 penados por los Juzgados de Violencia sobre la Mujer de Madrid, conformó la existencia de tres grupos: grupo de riesgo bajo (65\%), grupo de riesgo medio $(27,8 \%)$ y grupo de riesgo alto de violencia de género $(7,1 \%)$. Sugiere la posibilidad de una clasificación tipológica en tres grupos de agresores a partir de variables psicológicas diferenciales en la evaluación pre-tratamiento (personalidad límite, agresividad en general, agresividad hacia la pareja, consumo de alcohol, impulsividad etc.).

Los programas comunitarios no han considerado suficientemente las posibles tipologías en sus análisis. A pesar de ello, las características de los sujetos diagnosticadas en el plan empírico permiten ver al maltratador más allá de la imagen tópica y estereotipada que se viene difundiendo y posibilita que se dé un primer paso para integrar los programas dentro del abanico de respuestas orientadas a luchar contra la violencia de género ${ }^{32}$. Entre los beneficios de una clasificación tipológica, se destacan la predicción del riesgo de agresión hacia la pareja, la mayor o menor probabilidad de reincidencia en razón del subgrupo al que pertenezca el agresor y el diseño de protocolos de intervención específicos que cubran las necesidades concretas de los agresores ${ }^{33}$. En última instancia, una intervención con base en las tipologías permitiría profundizar los aspectos del programa que funcionen mejor para cada tipo de agresor ${ }^{34}$.

\subsection{Obligatoriedad.}

Entre los desafíos empíricos encontrados en los programas fuera de prisiones se destacan aquellos que poseen una relación directa con el marco legal español: la obligatoriedad de la

\footnotetext{
${ }^{29}$ BOIRA SARTO, Santiago; JODRÁ ESTEBAN, Pedro, "Psicopatología, características de la violencia y abandonos en programas para hombres violentos con la pareja: resultados en un dispositivo de intervención", Psicothema, Vol. 22, no 4 (2010), pp. 593-599. BOIRA SARTO, Santiago; TOMÁS-ARAGONÉS, Lucía, "Características psicológicas y motivación para el cambio en hombres condenados por violencia contra la pareja", International Journal of Psychological Research, Vol. 4, no 2 (2011), pp. 48-56.

${ }^{30}$ ECHEBURÚA/AMOR, "Perfil", cit. nota no 26, p. 119.

31 REDONDO RODRÍGUEZ, Natalia, Eficacia de un programa de tratamiento psicológico para maltratadores, Tesis Doctoral bajo supervisión de José Luis GRAÑA GÓMEZ, Universidad Complutense de Madrid, Facultad de Psicología, 2012.

${ }^{32}$ BOIRA/TOMÁS-ARAGONES, "Características”, cit. nota no 29, p. 49.

${ }^{33}$ REDONDO, Eficacia, cit. nota n ${ }^{\circ} 31$.

${ }^{34}$ LOINAZ/ECHEBURÚA, "Necesidades", cit. nota $n^{\circ}$ 9. LOINAZ CALVO, Ismael; ECHEBURÚA, Enrique; TORRUBIA, Rafael, "Tipología de agresores contra la pareja en prisión”, Psicothema, nº 22 (2010), pp. 106-111.
} 
Polít. crim. Vol. 10, № 19 (Julio 2015), Art. 10, pp. 297-317.

[http://www.politicacriminal.cl/Vol_10/n_19/Vol10N19A10.pdf]

intervención versus la gran resistencia al cambio por parte de los agresores. La decisión del hombre de acudir voluntariamente a un programa está condicionada por diversos factores ${ }^{35}$. El reconocimiento de que tienen un problema, de que están haciendo sufrir a otras personas, de que no pueden resolver la situación por sí solos y de que un cambio de actitud puede mejorar su nivel de bienestar o de su familia son algunas de las razones habituales que llevan al hombre a acudir a la consulta por primera vez ${ }^{36}$. Al lado de las razones personales -reconózcase que frecuentemente animadas por las mujeres parejas ${ }^{37}$ - se encuentra la orden judicial de participación en el programa. Esta incluso puede potenciar la resistencia al cambio, ya que algunos parecen tergiversar los hechos interpretando que su conducta no ha sido suficientemente grave para enviarles a prisión ${ }^{38}$.

La gravedad real de los hechos y la aceptación de la violencia son asumidas como puntos especialmente problemáticos, habida cuenta que los agresores suelen definir su comportamiento como "normal" o "aceptable" dentro de una relación de pareja; la reacción del sistema de justicia es interpretada como injusta ${ }^{39}$. La combinación de ambas circunstancias, concretamente la obligatoriedad en la participación y las distorsiones en la forma de atribuir las causas de los hechos por los que han sido condenados, hacen con que la motivación de los sujetos por participar y cambiar sea mínima ${ }^{40}$.

Asimismo la rehabilitación viene demostrándose posible a pesar de ser forzados a acudir al programa $^{41}$. Los profesionales que llevan a cabo este tipo de intervención justamente deben tener en cuenta que los agresores acuden obligatoriamente y que presentan como característica común negar, minimizar o atribuir la culpa de los hechos a otras personas/factores $^{42}$.

Para afrontar esta realidad, se vienen adoptando interesantes estrategias tanto para que el agresor se mantenga en el programa como para que se puedan promocionar los cambios deseados. En el País Vasco, por ejemplo, la motivación para mantener al sujeto en el tratamiento es lograda por medio de algunos argumentos persuasorios como sentirse mejor

\footnotetext{
${ }^{35}$ ECHEBURÚA, Enrique; CORRAL, Paz de, Manual de violencia familiar, Madrid: Siglo XXI, 1998.

${ }^{36}$ ECHEBURÚA, "Evaluación", cit. nota no 11. ECHEBURÚA/AMOR, "Perfil", cit. nota no 26.

${ }^{37}$ GELDSCHLÄGER, Heinrich; GINÉS, Oriol; PONCE, Älvaro, “Grupo psicoterapéutico con hombres que ejercen violencia de género”, en: ROCA CORTÉS, Neus; MASIP SERRA, Júlia (Eds.), Intervención Grupal en Violencia Sexista. Experiencia, Investigación y Evaluación, Barcelona: Herder, 2011. pp. 344-368, p. 345.

${ }^{38}$ MAGRO/HERNÁNDEZ/CUELLAR, “La aplicación”, cit. nota no 15, p. 201.

${ }^{39}$ LILA, "Predicting", cit. nota n ${ }^{\circ} 23$, p. 76. BOIRA, "Evaluación”, cit. nota no 12, p. 152.

40 ECHEBURÚA, "Evaluación”, cit. nota no 11. LILA, Marisol; HERRERO, Juan; GRACIA, Enrique, "Atribución de responsabilidad y minimización en hombres penados por violencia doméstica contra la mujer: un instrumento de evaluación.”, en: RODRÍGUEZ et al. (Eds.), Psicología Jurídica: Familia y victimología, Oviedo: Ediciones de la Universidad de Oviedo, 2008, pp. 271-279.

${ }^{4}$ CONCHELL/LILA/CATALÁ, "Cambios", cit. nota no 26. MAGRO/HERNÁNDEZ/CUELLAR, "La aplicación", cit. nota n ${ }^{\circ} 15$. BOIRA., "Intervención”, cit. nota $\mathrm{n}^{\mathrm{o}} 11$.

${ }^{42}$ BOIRA, "Características", cit. nota n ${ }^{\circ} 27$, pp. 148-153; SECRETARIA GENERAL DE INSTITUCIONES PENITENCIARIAS, Instrucción 10/2011 - Suspensión y Sustitución de condena de penas privativas de libertad. Especial referencia a la intervención con agresores por violencia de género en medidas alternativas - Anexo: Manual de Procedimiento. Gestión Administrativa. Metodología de Intervención en Violencia de Género, Servicio de Gestión Medidas y Penas Alternativas, Suspensiones y Sustituciones de Condena, $\mathrm{n}^{\circ} 2$, pp. 40 - 48, Disponible en http://www.institucionpenitenciaria.es/ [visitado el 08.04.2015].
} 
SORDI STOCK, Bárbara. "Programas de rehabilitación para agresores en España: un elemento indispensable de las políticas del combate a la violencia de género".

con la intervención, aprender a controlar las emociones, establecer una relación de pareja más adecuada, mejorar la autoestima etc. ${ }^{43}$ El Programa Contexto de Valencia apuesta con vigor por la entrevista motivacional ${ }^{44}$. Sugiere que la entrevista motivacional y la alianza terapéutica deben considerar la relación existente entre apoyo social, eventos vitales estresantes, ajuste psicológico (autoestima y depresión) del agresor y la atribución de culpa a la víctima ${ }^{45}$. Consiguientemente, trabajar tales cuestiones durante la intervención posibilitará que el sujeto alcance mejores resultados ${ }^{46}$. Ya Magro Servet, Hernández Ramos y Cuellar Otón $^{47}$ plantean que la medida más efectiva sería una intervención "obligatoria/voluntaria", es decir, que además del programa obligatorio por imperativo legal se le ofreciera un programa complementario a ser realizado voluntariamente tras finalizar el primero (obligatorio). Esta posibilidad contribuiría a hacer mínima la probabilidad de que el condenado simule un cambio que en la práctica puede estar no produciéndose. Destacan que en Alicante, alrededor de una cuarta parte de los penados que se dan de alta en el programa obligatorio acaban participando de las intervenciones voluntarias.

En una investigación cualitativa con profesionales responsables por programas, Boira, López y Tomás $^{48}$ han puesto en evidencia que algunos optan por una posición más confrontadora con el agresor y otros por una postura más pedagógica. Pese a estas diferencias, todos los profesionales han anotado que lo fundamental es "el encuadre de la intervención”. Así, cuestiones como el informar al agresor en qué consiste el grupo, cuáles son sus responsabilidades, qué se le va a exigir, cuánto dura el grupo, sus horarios etc., ayudan a definir los límites entre lo legal y lo psicológico.

La empatía del terapeuta y la presencia de expertos de ambos sexos también se mostraron útiles para el desarrollo de la intervención frente a los desafíos de un ambiente en el cual prevalece la resistencia y las respuestas hostiles. Tejerina y Martínez ${ }^{49}$ registraron los beneficios de terapeutas de ambos sexos al tiempo que destacaron la importancia del terapeuta poder flexibilizar la intervención en razón del perfil del grupo. La maleabilidad del programa según el grupo parece influir tanto en los instrumentos de evaluación sobre los resultados como en el interés de los sujetos por seguir el programa.

\footnotetext{
${ }^{43}$ ECHEBURÚA, “Adherencia”, cit. nota $\mathrm{n}^{\circ} 12$.

${ }^{44}$ CONCHELL/LILA/CATALÁ, "Cambios”, cit. nota no 26. LILA, Marisol; GRACIA, Enrique; HERRERO, Juan, "Asunción de responsabilidad en hombres maltratadores: Influencia de la autoestima, la personalidad narcisista y la personalidad antisocial”, Revista Latinoamericana de Psicología, Vol. 44, no 2 (2012), pp. 99108.

${ }^{45}$ LILA, Marisol; GRACIA, Enrique; MURGUI, Sergio, "Psychological adjustment and victim-blaming among intimate partner violence offenders: The role of social support and stressful life events", The European Journal of Psychology Applied to Legal Context, Vol. 5 (2013), pp. 147-153.

${ }^{46}$ LILA/GRACIA/HERRERO, “Asunción”, cit. nota n 44. LILA/GRACIA/MURGUI, "Psychological”, cit. nota $\mathrm{n}^{\mathrm{o}} 45$.

${ }^{47}$ MAGRO/HERNÁNDEZ/CUELLAR, "La aplicación”, cit. nota nº 15 .

${ }^{48}$ BOIRA SARTO, et al., "Evaluación cualitativa de un programa de intervención psicológica con hombres violentos dentro de la pareja.", Acciones e Investigaciones Sociales, Vol. 28 (2010), pp. 135-156, p. 148 y 150.

${ }^{49}$ TEJERINA/MARTINEZ, Evaluación, cit. nota $n^{\circ} 7$.
} 
Polít. crim. Vol. 10, № 19 (Julio 2015), Art. 10, pp. 297-317.

[http://www.politicacriminal.cl/Vol_10/n_19/Vol10N19A10.pdf]

Insistimos, pues, que al lado de la escasa y fluctuante motivación de los hombres de participar de un programa la negación o minimización de los hechos también sirve para afrontar el rechazo penal y social que este tipo de conducta suscita ${ }^{50}$. Prueba de ello es que la mayor visibilidad de la violencia de género en cuanto problema social no parece haber repercutido en el aumento de sujetos que participan voluntariamente ${ }^{51}$ de un tratamiento en medio comunitario ${ }^{52}$. No se puede obviar, por tanto, que el mando judicial y la consecuente amenaza de ingreso en prisión contribuyen a mantener al agresor en el tratamiento. Frente a este complejo escenario, consideramos fundamental recordar la importancia de establecer una relación terapéutica basada en la confianza con el sujeto y propiciar un ambiente exento de juicios moralizantes. En este sentido, Echeburúa y su equipo ${ }^{53}$ son contundentes al afirmar que "la motivación para el tratamiento es el motor de cambio y la piedra angular del éxito en un programa terapéutico con maltratadores". El objetivo que el profesional debe perseguir no se reduce a la motivación inicial del agresor, sino que debe estar direccionado a la motivación para mantener al sujeto en el tratamiento.

\subsection{Resultados.}

La eficacia (y/o efectividad) de los programas suele ser evaluada por medio de la reincidencia y del cambio terapéutico en los usuarios. En otras palabras, entre los resultados de los programas se contemplan no solo nuevos episodios de violencia sino qué variables relacionadas con la conducta violenta parecen haber sido objeto de mudanza. A día de hoy parece haber un cierto consenso que el compromiso del terapeuta es generar expectativas de cambio realistas ${ }^{54}$. La práctica ha demostrado que determinadas conductas, como ira y celos son muy complejas de eliminar por completo, si bien el sujeto puede aprender a mantenerlas bajo control y a canalizarlas de una forma adecuada ${ }^{55}$.

\footnotetext{
${ }^{50}$ ECHEBURÚA/AMOR, "Perfill”, cit. nota n 26.

${ }^{51}$ Entre las técnicas que garantizan la motivación de los participantes en los programas voluntarios se destacan las llamadas de retención proactiva y apoyo social (proactive retention and social support), como las implementadas en Cataluña. La policía autonómica llevó a cabo, junto con el Instituto de Reinserción Social (IReS), un proyecto innovador y que contó con la aplicación de técnicas de retención proactiva y apoyo en aquellos hombres denunciados por violencia contra la pareja. Estas técnicas motivacionales fueron aplicadas por los propios agentes de la policía, quienes tenían por misión informar y motivar a los hombres implicados en episodios violentos sobre los recursos existentes en su zona geográfica en cuanto a tratamiento de agresores (concretamente el programa SAHM - Servicio de Atención para Hombres Maltratadores ofrecido por el IReS en la provincia de Gerona), mediante llamadas telefónicas y entrevistas personalizadas. El objetivo era que los agresores participasen activamente en el programa y que se pudiera realizar un seguimiento (retención proactiva y apoyo) previamente a su incorporación al programa o durante el inicio del mismo. Recientemente, se analizó una muestra de 142 hombres que habían acudido de forma voluntaria y gratuita al SAHM (años 2001 - 2008) y los resultados han sido estadísticamente significativos con respecto al grupo de hombres al que se ha aplicado técnicas de retención proactiva y apoyo en relación a la adherencia terapéutica al programa. Véase SUBIRANA-MALARET, Montse; ANDRÉS-PUEYO, Antonio, "Retención proactiva y adherencia terapéutica en programas formativos para hombres maltratadores de la pareja", Psychosocial Intervention, Vol. 22, nº 02 (2013), pp. 95-104.

${ }^{52}$ BOIRA, Hombres, cit. nota $\mathrm{n}^{\circ} 2$.

${ }^{53}$ ECHEBURÚA, "Evaluación”, cit. nota no 11, p. 215.

${ }^{54}$ PÉREZ/MARTÍNEZ, "Evaluación”, cit. nota n ${ }^{\circ} 16$.

${ }^{55}$ ECHEBURÚA, "Evaluación”, cit. nota n' 11.
} 
SORDI STOCK, Bárbara. "Programas de rehabilitación para agresores en España: un elemento indispensable de las políticas del combate a la violencia de género".

Desde una perspectiva holística, afirmamos que las evaluaciones publicadas en España son bastante optimistas. No obstante, los resultados de los programas como pena/medida alternativa a la prisión no ofrecen datos concluyentes. Veamos.

La evaluación del PRIA en medio abierto revela que "se ha producido un cambio terapéutico significativo en los usurarios como consecuencia del tratamiento",56. El programa parece haber incidido en las variables relacionadas con la génesis de la violencia, una vez que tras finalizar la intervención los hombres manifestaron, entre otras cuestiones, menos conflictos con la pareja, menos actitud sexista, menos celos, mayor asunción de responsabilidad, menos ira y más control de sus emociones. Si de una parte la ausencia de factores de riesgo entre el grupo mayoritario de usuarios ha resultado en un mayor impacto del tratamiento, de otra parte el programa también logró un efecto positivo en el minoritario "grupo de riesgo". Ya sobre la reincidencia se observó que apenas el 4,6\% de los usuarios (29 sujetos) volvió a tener una nueva denuncia policial después de finalizado el tratamiento (período de seguimiento de 1 año).

En Cataluña, Pérez Ramírez y Martínez García ${ }^{57}$ partieron de dos muestras de agresores, aunque todos los programas fueron evaluados conjuntamente. Una primera apreciación pre/post-tratamiento con una muestra de 93 sujetos indica que tras el programa los hombres -en general- manifestaron menos conflictos con la pareja, mayor satisfacción con la pareja, mejor consciencia de su problema, menos ira, más control de sus emociones y menos problema de alcoholismo. En un segundo orden de consideraciones, tras un seguimiento de 12 meses (medida) con una muestra de 170 sujetos se constató que apenas el 8,8\% (15 individuos) tuvieron una nueva denuncia policial. Entre ellos, el 6,4\% fue por un nuevo delito de violencia de género y el $2,4 \%$ por otros delitos, como robos y lesiones. Así, el $92 \%$ de los agresores que han finalizado un programa de rehabilitación en medio comunitario y derivados por la Justicia Penal en Cataluña parece que no han vuelto a reincidir, o por lo menos no se les ha denunciado ante la policía en un tiempo medio de 12 meses. Frente a estos datos concluyeron las investigadoras que los programas realizados por distintas entidades en Cataluña poseen "efectividad moderada".

En Zaragoza, el índice de reincidencia verificado en un período de seguimiento de 18 meses fue considerado "bajo" por Boira y compañeros ${ }^{58}$. Únicamente el 6,38\% (3 sujetos) de los agresores que realizaron un programa fueron detenidos tras su finalización. Los demás manifestaron no haber tenido algún incidente de violencia con la víctima, dato este confirmado por medio de un rastreo en la base de datos del Sistema de Seguimiento Integral en los casos de Violencia de Género (Sistema VdG), gestionada el Ministerio del Interior.

\footnotetext{
${ }^{56}$ SECRETARIA GENERAL DE INSTITUCIONES PENITENCIARIAS, Evaluación, cit. nota n ${ }^{\circ}$ 17, p. 55.

${ }^{57}$ PÉREZ/MARTÍNEZ, "Evaluación”, cit. nota no 16. PÉREZ RAMÍREZ, Meritxell; MARTÍNEZ GARCÍA, Marian, La reincidencia de los condenados por delitos de violencia de género en programas formativos aplicados desde la ejecución penal en la comunidad, Generalitat de Cataluña, Centre d' Estudis Jurídics i Formació Especialitzada, 2010.

${ }^{58}$ BOIRA, “Intervención”, cit. nota nº 11, p. 25.
} 
Polít. crim. Vol. 10, No 19 (Julio 2015), Art. 10, pp. 297-317.

[http://www.politicacriminal.cl/Vol_10/n_19/Vol10N19A10.pdf]

En Alicante, la reincidencia del $10 \%$ post-tratamiento fue considera "muy aceptable" por Magro Servet, Hernández Ramos y Cuellar Otón ${ }^{59}$. Ningún de los nuevos actos de violencia fueron de lesión grave o resultaron en muerte de la víctima.

En Madrid, las tasas de reincidencia post-tratamiento encontradas para las distintas tipologías según Redondo Rodríguez ${ }^{60}$ no han sido significativas. Concretamente, del 5,3\% para el grupo de agresores considerado de riesgo alto; del 5,4\% para el grupo de riesgo medio y del $4 \%$ para grupo de riesgo bajo. No obstante, si se compara aquellos que recibieron tratamiento y el grupo control, la tasa de reincidencia es del 4,8\% para tratados frente al 6,8\% para no tratados. Los hallazgos sugieren que el tratamiento tiene un efecto positivo aunque limitado, al tiempo que indica que el hecho de estar enfrentando un proceso judicial activo parece tener un peso a la hora de disminuir la reincidencia.

Los resultados del programa de Valencia apuntan en buen sentido según los estudios de Conchell Diranzo, Lila y Catalá Miñana ${ }^{61}$. Los hombres que finalizaron el programa se mostraron, entre otras cuestiones, más responsables de sus actos, menos tolerantes con la violencia de género y menos proclives a utilizar la violencia como una forma para resolver sus conflictos, factor último que insinúa una disminución de la reincidencia futura. Una cuestión muy interesante que también ha sido evaluada se relaciona con el apoyo comunitario percibido por los penados tras cumplimentar la intervención. A pesar de que la evaluación post-tratamiento no indica un cambio significativo en variables como la integración, apoyo informal y apoyo formal, parece ser que hubo una mejora de las mismas tras finalizar el programa, diagnóstico que sugiere la necesidad de seguir trabajando con las redes sociales de los sujetos.

Por último, el estudio de Arrigoni et al. ${ }^{62}$, a pesar de referirse a una pequeña muestra de penados (38 sujetos), también sugiere que un programa desarrollado en Andalucía incide positivamente a respecto de las ideas distorsionadas sobre la mujer y en el uso de la violencia como forma de resolución de conflictos. No obstante, destacan que si bien la evaluación pre/post-tratamiento demostró dichos impactos en el 65\%, de los agresores hay un importante porcentual, concretamente el 35\%, que no presentó cambio en relación a ambas cuestiones. Es más, parece que en algunos de estos penados incluso se acentuaron sus distorsiones cognitivas tras la intervención.

Lo que pretendemos destacar con el conjunto de investigaciones citadas es que en determinados sujetos la intervención ejerce un impacto positivo sobre ciertos factores asociados a la violencia hacia la mujer y muchos de los penados no vuelven a cometer nuevos delitos tras participaren de un programa específico. Aunque la prudencia exija una reflexión más serena sobre hasta qué punto son los componentes del tratamiento los que realmente han provocado un cambio en el agresor constatables en la evaluación posttratamiento, lo cierto es que cuando se ha logado realizar seguimiento un importante

\footnotetext{
${ }^{59}$ MAGRO/HERNÁNDEZ/CUELLAR, “La aplicación”, cit. nota nº 15, p. 200.

${ }^{60}$ REDONDO, Eficacia, cit. nota n ${ }^{\circ} 31$, pp. 266-267.

${ }^{61}$ CONCHELL/LILA/CATALÁ, "Cambios", cit. nota n' 26.

${ }^{62}$ ARRIGONI, “Aplicación”, cit. nota n 25.
} 
SORDI STOCK, Bárbara. "Programas de rehabilitación para agresores en España: un elemento indispensable de las políticas del combate a la violencia de género".

porcentaje de sujetos tratados en medio comunitario no recurrió al uso de la violencia ${ }^{63}$. Por tanto, antes que defender la existencia de un cambio terapéutico definitivo es preferible mantener más bien una postura sensata sobre los posibles aprendizajes a atribuir a los programas, destacándose el uso de la violencia como una estrategia válida para hacer frente a los problemas ${ }^{64}$.

Particularmente sobre la reincidencia, las únicas investigaciones que cuentan con seguimiento indican índices, en nuestra opinión, aceptables. Concretamente el 4,4\% ${ }^{65}$, el $8,8 \%{ }^{66}$ y el $6,38 \%^{67}$. No se puede descartar asimismo que quizás estos índices estén vinculados al bajo riesgo que presentan los agresores condenados a una pena/media alternativa, justamente lo que posibilita que ellos participen de un programa en medio abierto $^{68}$.

Para no contar con la suerte o fatalidad de dicha sospecha, la responsabilidad científica avala la pertinencia de identificar variables que vienen demostrándose íntimamente relacionadas con la reincidencia y consiguientemente con los demás índices de éxitos del tratamiento $^{69}$. En una primera línea debe subrayarse el estar bajo efecto de alguna droga/alcohol durante la comisión del delito y el haber sido testimonio de malos tratos en la infancia, cuestiones estas que pueden ser diagnosticadas en la fase inicial del programa y abordadas por medio de otros programas de apoyo complementarios al programa principal de violencia de género ${ }^{70}$. Consiguientemente, es posible que se recomiende al sujeto realizar una doble intervención que pueda lidiar simultáneamente con el consumo abusivo de alcohol/droga y la gestión de la violencia por ejemplo ${ }^{71}$.

Al lado de las mencionadas variables, vienen ganando acogida otras paulatinamente, si bien carecen de mayor profundidad de análisis. Igualmente se han mostrado relacionadas con la reincidencia la duración de la pena, la ausencia de una estructura de apoyo social adecuado para hombres que ejercen violencia ${ }^{72}$ y el estar en paro $^{73}$. Frente a este contexto, y teniendo en cuenta que no son pocos los casos en los que la relación entre víctima y agresor no se rompe tras una condena, las investigaciones empíricas empiezan a concentrar esfuerzos en identificar las variables psicosociales que predicen los indicadores de éxito de un

\footnotetext{
${ }^{63}$ Téngase como ejemplo ECHEBURÚA, “Evaluación”, cit. nota n 11 . BOIRA, "Intervención”, cit. nota no 11.

${ }^{64}$ PÉREZ, "Evaluación”, cit. nota no 14.

${ }^{65}$ SECRETARIA GENERAL DE INSTITUCIONES PENITENCIARIAS, Evaluación, cit. nota n 17.

${ }^{66}$ PÉREZ/MARTÍNEZ, "Evaluación”, cit. nota n 16.

${ }^{67}$ BOIRA, "Intervención”, cit. nota n 11.

${ }^{68}$ PÉREZ/MARTÍNEZ, "Evaluación”, cit. nota no 16.

${ }^{69}$ LILA, "Predicting", cit. nota n' 23. LILA, Marisol et al., "Valoración del riesgo de reincidencia en violencia contra la mujer en las relaciones de pareja: importancia del apoyo social.”, Revista de Psicología Social, Vol. 28, no 2 (2013), pp. 225-236.

${ }^{70}$ PÉREZ/MARTÍNEZ, "Evaluación”, cit. nota nº 16.

${ }^{71}$ BOIRA/JODRA, "Psicopatología", cit. nota n' 29. LILA, "Valoración”, cit. nota no 69.

${ }^{72}$ LILA, "Valoración", cit. nota ${ }^{\circ} 69$.

${ }^{73}$ PÉREZ/MARTÍNEZ, "Evaluación”, cit. nota no 16.
} 
Polít. crim. Vol. 10, Nº 19 (Julio 2015), Art. 10, pp. 297-317.

[http://www.politicacriminal.cl/Vol_10/n_19/Vol10N19A10.pdf]

programa $^{74}$ y en la reevaluación de los instrumentos de predicción de riesgo ${ }^{75}$ bien como de reincidencia ${ }^{76}$.

\subsubsection{Los desafíos en evaluar los resultados.}

Pese al amplio abanico de estudios publicados en España, continúa existiendo una gran dificultad para evaluar adecuadamente los resultados de los programas. Las razones son varias, destacándose los pocos instrumentos utilizados y validados en el ámbito internacional que se pueden aplicar a la realidad española y los auto informes como estrategia principal en la evaluación del cambio en el penado ${ }^{77}$.

Por consiguiente, conductas como el maltrato psicológico pueden seguir estando presentes aunque la violencia física haya cesado y la víctima no haya presentado nueva denuncia ${ }^{78}$. Además, algunos hombres suelen mostrar ante el terapeuta una "doble fachada"79 y falsean las pruebas de cambio de comportamiento ${ }^{80}$. Si bien para afrontar esta cuestión se apuesta en el uso de herramientas que permitan a los profesionales a obtener indicadores del grado de la asunción de la responsabilidad al principio y a lo largo de la intervención ${ }^{81}$, la comunidad científica todavía no trabaja bajo sólidos instrumentos capaces de dar una respuesta adecuada a dicha problemática ${ }^{82}$.

\section{2. ¿Y las mujeres víctimas?}

El único dato quizás incuestionable que se puede extraer de las investigaciones con agresores publicadas en España es que existe un porcentaje alto de víctimas que sigue bien manteniendo relación o bien manteniendo algún tipo de contacto con el usuario de un programa. Específicamente, en Cataluña el $13 \%{ }^{83}$, en Alicante el $30 \%{ }^{84}$, en Aragón el

\footnotetext{
${ }^{74}$ ECHEBURÚA, Enrique et al., "Terapias Psicológicas Basadas en la Evidencia: Limitaciones y Retos de Futuro", Revista Argentina de Clínica Psicológica, Vol. XIX (2010), pp. 247- 256. LILA, "Valoración”, cit. nota $n^{\circ} 69$.

${ }^{75}$ El instrumento SARA es el que se encuentra más extendido en España. Para una discusión más a fondo sobre la predicción del riesgo consultar ANDRÉS-PUEYO, Antonio; ECHEBURÚA, Enrique, "Valoración del riesgo de violencia: instrumentos disponibles e indicaciones de aplicación", Psicothema, Vol. 22, n 3 (2010), pp. 403-409.

${ }^{76}$ ECHEBURÚA, Enrique et al., "Assessing risk markers in intimate partner femicide and severe violence. A new assessment instrument”, Journal of Interpersonal Violence, Vol. 24 (2009), pp. 925-939. LILA, "Valoración", cit. nota n 69.

77 CARBAJOSA, Pablo; BOIRA, Santiago, "Estado actual y retos futuros de los programas para hombres condenados por violencia de género en España”, Psychosocial Intervention, no 22 (2013), pp. 145-152. SECRETARIA GENERAL DE INSTITUCIONES PENITENCIARIAS, Evaluación, cit. nota n ${ }^{\circ} 17$, p. 57. TEJERINA/MARTÍNEZ, Evaluación, cit. nota nº 5, p. 35.

${ }^{78}$ ECHEBURÚA, Enrique; FERNÁNDEZ-MONTALVO, Javier; DE LA CUESTA, José Luis, “Articulación de medidas penales y de tratamiento psicológico en los hombres violentos en el hogar", Psicopatología Clínica, Legal y Forense, Vol. 1, nº 2 (2001), pp. 19-31.

${ }^{79}$ ECHEBURÚA, "Evaluación”, cit. nota $\mathrm{n}^{\circ} 11$.

${ }^{80}$ BOIRA/TOMÁS-ARAGONES, “Características”, cit. nota n 29.

${ }^{81}$ LILA/HERRERO/GRACIA, “Atribución”, cit. nota n' 40.

${ }^{82}$ CARBAJOSA/BOIRA, "Estado", cit. nota n 77.

${ }^{83}$ PÉREZ/MARTÍNEZ, "Evaluación”, cit. nota no 16.

${ }^{84}$ MAGRO/HERNÁNDEZ/CUELLAR, “La aplicación”, cit. nota no 15 .
} 
SORDI STOCK, Bárbara. "Programas de rehabilitación para agresores en España: un elemento indispensable de las políticas del combate a la violencia de género".

$34,88 \%^{85}$, en Valencia el $17,9 \%^{86}$ en Madrid el $14,8 \%{ }^{87}$ y el $26 \%$ para el resto de España ${ }^{88}$. Por ello, consideramos fundamental que el hombre que inicie un tratamiento bajo determinación judicial lo haga dentro de un abordaje integral dónde se facilite a la mujer víctima el acceso a la asistencia psicológica, jurídica y social de forma paralela e independiente de la estructura ofrecida al agresor.

Los hombres que cumplen pena/medida alternativa no cuentan con un factor de inocuización como es el ingreso en prisión. Ante el deseo de confiar en la eficacia de los programas como respuesta del Sistema de Justicia penal, no podemos obviar que se encuentra en juego la seguridad de las víctimas. Ahora bien, este escenario no implica obviar las redes sociales y los sistemas de apoyo comunitario también para agresores, ya que el entorno de aquellos que son o han sido usuarios de un programa puede favorecer el uso de la violencia y la percepción sobre su gravedad ${ }^{89}$. La absoluta necesidad de ejecutar medidas penales firmes y contundentes no excluye el paralelo desarrollo de medidas complementarias de intervención psicosocial y la profundización en los mecanismos de actuación con los agresores ${ }^{90}$.

\section{Conclusión}

La experiencia española confirma, aun cuando sigan existiendo desafíos de diversos órdenes, que los programas de rehabilitación para agresores constituyen un elemento indispensable de las políticas públicas de combate a la violencia de género en razón de los efectos preventivos especiales que presentan. Nuestro estudio ha demostrado que en este momento se discute cómo rehabilitar y no si es posible rehabilitar.

Entre las circunstancias que han contribuido para el cambio de matiz, quisiera reforzar la apuesta por la mejora en la calidad de las intervenciones proporcionada por la proliferación de investigaciones realizadas por distintos grupos. La evidencia científica ha permitido el avance y la consecuente sofisticación de los programas para agresores de violencia de género $^{91}$. Todo ello porque la ausencia de pruebas sobre su capacidad de reducir la violencia supone un desperdicio de recursos públicos y un aumento de los riesgos de nuevas victimizaciones ${ }^{92}$. La financiación debe estar apenas destinada a los programas que hayan demostrado resultados positivos ${ }^{93}$.

Como reflexión final subrayo que ubicar los programas como estrategias de prevención terciaria es percibir que estos pueden ser una respuesta judicial que contribuye a reducir el problema social de la violencia hacia la mujer, siempre y cuando sean trabajados desde un

\footnotetext{
${ }^{85}$ BOIRA, "Intervención”, cit. nota no 11.

${ }^{86}$ LILA, "Valoración", cit. nota $n^{\circ} 69$.

${ }^{87}$ REDONDO, Eficacia, cit. nota n 31 .

${ }^{88}$ SECRETARIA GENERAL DE INSTITUCIONES PENITENCIARIAS, Evaluación, cit. nota no 17.

${ }^{89}$ GRACIA, Enrique; HERRERO, Juan, "Public attitudes toward reporting partner violence against women and reporting behavior.”, Journal of Marriage and Family, Vol. 68 (2006), pp. 759-768.

${ }^{90}$ GELDSCHLÄGER, “Grupo", cit. nota n 35.

${ }^{91}$ CARBAJOSA, "Estado", cit. nota $\mathrm{n}^{\mathbf{0}} 75$.

92 GELDSCHLÄGER/GINÉS/PONCE, “Grupo", cit. nota n 37.

${ }^{93}$ ECHEBURÚA, “Evaluación”, cit. nota n ${ }^{\circ} 11$.
} 
Polít. crim. Vol. 10, № 19 (Julio 2015), Art. 10, pp. 297-317.

[http://www.politicacriminal.cl/Vol_10/n_19/Vol10N19A10.pdf]

matiz interdisciplinar y estén fundamentados en la evidencia científica. En otras palabras, una política criminal que comprenda la complejidad y las especificidades de la violencia de género patrocina los programas vinculados a la Justicia Penal porque son la mejor solución que se ha encontrado y no porque sean la solución para esta clase de delitos. 
SORDI STOCK, Bárbara. "Programas de rehabilitación para agresores en España: un elemento indispensable de las políticas del combate a la violencia de género".

\section{BIBLIOGRAFÍA}

ANDRÉS-PUEYO, Antonio; ECHEBURÚA, Enrique, "Valoración del riesgo de violencia: instrumentos disponibles e indicaciones de aplicación", Psicothema, Vol. 22, n 3 (2010), pp. 403-409.

ARRIGONI et al., "Aplicación de un programa terapéutico en hombres violentos contra la pareja", Anuario de Psicología Jurídica, no 23 (2013), pp. 3-9.

BOIRA SARTO, Santiago et al., "Evaluación cualitativa de un programa de intervención psicológica con hombres violentos dentro de la pareja.", Acciones e Investigaciones Sociales, Vol. 28 (2010), pp. 135-156.

BOIRA SARTO, Santiago et al., "Intervención psicológica en la comunidad en hombres condenados por violencia de género", Anales de Psicología, Vol. 29, nº 1 (2013), pp.19-28.

BOIRA SARTO, Santiago, Hombres Maltratadores. Historias de Violencia Masculina, Zaragoza: Prensas Universitarias de Zaragoza, 2010.

BOIRA SARTO, Santiago; JODRÁ ESTEBAN, Pedro, "Psicopatología, características de la violencia y abandonos en programas para hombres violentos con la pareja: resultados en un dispositivo de intervención", Psicothema, Vol. 22, n 4 (2010), pp. 593-599.

BOIRA SARTO, Santiago; TOMÁS-ARAGONÉS, Lucía, “Características psicológicas y motivación para el cambio en hombres condenados por violencia contra la pareja", International Journal of Psychological Research, Vol. 4, nº 2 (2011), pp. 48-56.

CARBAJOSA, Pablo; BOIRA, Santiago, "Estado actual y retos futuros de los programas para hombres condenados por violencia de género en España", Psychosocial Intervention, $\mathrm{n}^{\mathrm{o}} 22$ (2013), pp. 145-152.

CONCHELL DIRANZO, Raquel; LILA MURILLO, Marisol; CATALÁ MIÑANA, Alba, "Cambios psicosociales en un programa de intervención con hombres penados por violencia contra la mujer.", Revista de Psicología de la Universidad de Chile, Vol. 21, no 2 (2012), pp. $159-185$.

ECHAURI TIJERAS, José Antonio, "Efectividad de un programa de tratamiento con inmigrantes que ejercen violencia de género contra la pareja", Psicothema, Vol. 25, $\mathrm{n}^{\circ} 1$ (2013), pp. 49-54.

ECHEBURÚA, Enrique et al., "Evaluación de la eficacia de un tratamiento cognitivoconductual para hombres violentos contra la pareja en un marco comunitario: Una experiencia de 10 años (1997-2007)", International Journal of Clinical and Health Psychology, no 9 (2009), pp. 199-217.

ECHEBURÚA, Enrique et al., "Terapias Psicológicas Basadas en la Evidencia: Limitaciones y Retos de Futuro", Revista Argentina de Clínica Psicológica, Vol. XIX (2010), pp. 247- 256.

ECHEBURÚA, Enrique, "Adherencia al tratamiento en hombres maltratadores contra la pareja en un entorno comunitario: Realidad actual y retos de futuro", Psychosocial Intervention, $\mathrm{n}^{\circ} 22$ (2013), pp. 87-93.

ECHEBURÚA, Enrique; AMOR, Pedro Javier, "Perfil psicopatológico e intervención terapéutica con los agresores contra la pareja”, Revista Española de Medicina Legal, Vol. 36, no 3 (2010), pp. 117-121. 
Polít. crim. Vol. 10, No 19 (Julio 2015), Art. 10, pp. 297-317.

[http://www.politicacriminal.cl/Vol_10/n_19/Vol10N19A10.pdf]

ECHEBURÚA, Enrique; CORRAL, Paz de, Manual de violencia familiar, Madrid: Siglo XXI, 1998.

ECHEBURÚA, Enrique; FERNÁNDEZ-MONTALVO, Javier; DE LA CUESTA, José Luis, "Articulación de medidas penales y de tratamiento psicológico en los hombres violentos en el hogar", Psicopatología Clínica, Legal y Forense, Vol. 1, nº 2 (2001), pp. 19-31.

EXPÓSITO, Francisca; RUIZ, Sergio, "Reeducación de Maltratadores: Una Experiencia de Intervención desde la Perspectiva de Género", Intervención Psicosocial, Vol. 19, n 2 (2010), pp. 145-151.

FERNÁNDEZ-MONTALVO, Javier et al., "Violencia de género e inmigración: perfil diferencial de hombres maltratadores nacionales e inmigrantes", Psicología Conductual, Vol. 19, nº 2 (2011), pp. 439-452.

GELDSCHLÄGER, Heinrich; GINÉS, Oriol; PONCE, Älvaro, “Grupo psicoterapéutico con hombres que ejercen violencia de género", en: ROCA CORTÉS, Neus; MASIP SERRA, Júlia (Eds.), Intervención Grupal en Violencia Sexista. Experiencia, Investigación y Evaluación, Barcelona: Herder, 2011. pp. 344-368.

GRACIA, Enrique; HERRERO, Juan, "Public attitudes toward reporting partner violence against women and reporting behavior.", Journal of Marriage and Family, Vol. 68 (2006), pp. 759-768.

LILA, Marisol et al., "Cuando las cifras hablan: Programa de rehabilitación para maltratadores e inmigración", ca. 2011 Disponible en http://www.uv.es/contexto/enriquegracia/Investigacion.htm [visitado el 08.04.2015].

LILA, Marisol et al., "Predicting Success Indicators of an Intervention Programme for Convicted Intimate-Partner Violence Offenders: the Contexto Programme.”, The European Journal of Psychology Applied to Legal Context, Vol. 5, no 1 (2013), pp. 73-95.

LILA, Marisol et al., "Valoración del riesgo de reincidencia en violencia contra la mujer en las relaciones de pareja: importancia del apoyo social.", Revista de Psicología Social, Vol. 28, no 2 (2013), pp. 225-236.

LILA, Marisol; CONCHELL, Raquel, "Programa CONTEXTO: programa de Intervención para maltratadores en la provincia de Valencia.", ReCrim (2009), pp.199-215.

LILA, Marisol; GARCÍA, António; LORENZO, María Victória, Manual de Intervención con Maltratadores, Valencia: Universitat de València, 2010.

LILA, Marisol; GRACIA, Enrique; HERRERO, Juan, "Asunción de responsabilidad en hombres maltratadores: Influencia de la autoestima, la personalidad narcisista y la personalidad antisocial", Revista Latinoamericana de Psicología, Vol. 44, $\mathrm{n}^{\mathrm{o}} 2$ (2012), pp. 99-108.

LILA, Marisol; GRACIA, Enrique; MURGUI, Sergio, "Psychological adjustment and victim-blaming among intimate partner violence offenders: The role of social support and stressful life events.", The European Journal of Psychology Applied to Legal Context, Vol. 5 (2013), pp. 147-153.

LILA, Marisol; HERRERO, Juan; GRACIA, Enrique, "Atribución de responsabilidad y minimización en hombres penados por violencia doméstica contra la mujer: un instrumento de evaluación.", en: RODRÍGUEZ et al. (Eds.), Psicología Jurídica: Familia y victimología, Oviedo: Ediciones de la Universidad de Oviedo, 2008, pp. 271-279. 
SORDI STOCK, Bárbara. "Programas de rehabilitación para agresores en España: un elemento indispensable de las políticas del combate a la violencia de género".

LOINAZ CALVO, Ismael, Aproximación teórica y empírica al estudio de las tipologías de agresores de pareja: análisis descriptivo de variables e instrumentos de evaluación en el centro penitenciario Brians-2, Madrid: Ministerio del Interior, Secretaria General Técnica, 2009.

LOINAZ CALVO, Ismael; ECHEBURÚA, Enrique, "Necesidades terapéuticas en agresores de pareja según su perfil diferencial", Clínica Contemporánea, Vol. 1, n 2 (2010), pp. 85-95.

LOINAZ CALVO, Ismael; ECHEBURÚA, Enrique; TORRUBIA, Rafael, “Tipología de agresores contra la pareja en prisión", Psicothema, no 22 (2010), pp. 106-111.

LÓPEZ SAMANIEGO, Luz, "Salud y condena por violencia de género. (Asistentes a programas formativos de reeducación)", La Ley Penal: revista de Derecho penal, procesal y penitenciario, $\mathrm{n}^{\circ} 93$ (2012), pp. $94-103$.

MAGRO SERVET, Vicente; HERNÁNDEZ RAMOS, Carmelo; CUELLAR OTÓN, Pablo, "La aplicación de programas formativos de reeducación para condenados por delitos relacionados con la violencia de género, en aplicación de las medidas de suspensión de la ejecución de la pena: el programa de la audiencia provincial de alicante", en: MARTÍNEZ GARCÍA, Elena; VEGAS AGUILAR, Juan Carlos, La ejecución de los trabajos en beneficio de la comunidad por delitos de violencia de género, Tirant Monografías 763, Valencia: Tirant lo Blanch, 2012, pp. 172 - 207.

PÉREZ RAMÍREZ, Meritxell; MARTÍNEZ GARCÍA, Marian, "Evaluación de los programas formativos aplicados desde la ejecución penal en la comunidad para delitos de violencia de género", en: CENTRE D' ESTUDIS JURÍDICS I FORMACIÓ ESPECIALITZADA, Intervención con agresores de violencia de género. Evaluación de los programas formativos aplicados desde la ejecución penal en la comunidad para delios de violencia de género. Clasificación de agresores de pareja en prisión. Implicaciones terapéuticas y de gestión del riesgo, Justicia y Sociedad, no 34, Centre d' Estudis Jurídics i Formació Especialitzada: 2011, pp. 11 152.

PÉREZ RAMÍREZ, Meritxell; MARTÍNEZ GARCÍA, Marian, La reincidencia de los condenados por delitos de violencia de género en programas formativos aplicados desde la ejecución penal en la comunidad, Generalitat de Cataluña, Centre d' Estudis Jurídics i Formació Especialitzada, 2010.

REDONDO RODRÍGUEZ, Natalia, Eficacia de un programa de tratamiento psicológico para maltratadores, Tesis Doctoral bajo supervisión de José Luis GRAÑA GÓMEZ, Universidad Complutense de Madrid, Facultad de Psicología, 2012.

RUIZ, Sergio; EXPÓSITO, Francisca, "Intervención con hombres en suspensión condicional de condena por violencia de género", Anuario de Psicología Jurídica, Vol. 18 (2008), pp. 81- 89.

SECRETARIA GENERAL DE INSTITUCIONES PENITENCIARIAS - INSTITUTO DE CIENCIAS FORENSES Y DE LA SEGURIDAD, Evaluación del Programa. "Violencia de Género: programa de intervención para agresores", en medidas alternativas, Madrid: ca. 2012. Disponible en http://www.institucionpenitenciaria.es/ [visitado el 08.04.2015].

SECRETARIA GENERAL DE INSTITUCIONES PENITENCIARIAS, Documentos Penitenciarios 2. Programa de Tratamiento en Prisión para agresores en el ámbito 
Polít. crim. Vol. 10, № 19 (Julio 2015), Art. 10, pp. 297-317.

[http://www.politicacriminal.cl/Vol_10/n_19/Vol10N19A10.pdf]

familiar. Grupo de trabajo sobre violencia de género, Madrid: 2005. Disponible en http://www.institucionpenitenciaria.es/[visitado el 08.04.2015]

SECRETARIA GENERAL DE INSTITUCIONES PENITENCIARIAS, Documentos Penitenciarios 7. Violencia de Género. Programa de Intervención para Agresores PRIA, Madrid: 2010. Disponible en http://www.institucionpenitenciaria.es/ [visitado el 08.04.2015].

SECRETARIA GENERAL DE INSTITUCIONES PENITENCIARIAS, El Delito de Violencia de Género y los Penados Extranjeros anexo al Documentos Penitenciarios 7. Violencia de Género. Programa de Intervención para Agresores. PRIA, Madrid: 2011, Disponible en http://www.institucionpenitenciaria.es/ [visitado el 08.04.2015].

SECRETARIA GENERAL DE INSTITUCIONES PENITENCIARIAS, Instrucción 10/2011 - Suspensión y Sustitución de condena de penas privativas de libertad. Especial referencia a la intervención con agresores por violencia de género en medidas alternativas - Anexo: Manual de Procedimiento. Gestión Administrativa. Metodología de Intervención en Violencia de Género, Servicio de Gestión Medidas y Penas Alternativas, Suspensiones y Sustituciones de Condena, $\mathrm{n}^{\mathbf{o}} 2$, Disponible en http://www.institucionpenitenciaria.es/ [visitado el 08.04.2015].

SORDI STOCK, Bárbara, Análisis político criminal de los programas de rehabilitación para agresores de violencia de género, Tesis Doctoral bajo supervisión de Borja MAPELLI CAFFARENA, Universidad de Sevilla, Departamento de Derecho Penal y Procesal, 2014.

SORDI STOCK, Bárbara, “¿Nuevos horizontes? en los programas de rehabilitación para agresores de violencia de género", InDret - Revista para el análisis del Derecho, 2015.

SUBIRANA-MALARET, Montse; ANDRÉS-PUEYO, Antonio, "Retención proactiva y adherencia terapéutica en programas formativos para hombres maltratadores de la pareja", Psychosocial Intervention, Vol. 22, no 02 (2013), pp. 95-104.

TEJERINA, Benjamín; MARTÍNEZ, María, Evaluación de la implementación de programas de intervención re-educativa con agresores en el ámbito de la violencia de género, Informes del Centro de Estudios sobre la Identidad Colectiva C.E.I.C/IKI: 2011. 